\title{
Serum Homocysteine Level in Patients with Cerebral Venous Sinus Thrombosis (CVST): Relation to Initial Thrombosis Severity and Outcome M.A.Al Baklawy, R.M.Khodair, M.K.Faheem and I.A.Abd Elrassoul \\ Ophthalmology Dept., Faculty of Medicine, Benha Univ., Benha, Egypt E-Mail: M.Baklawy@gmail.com
}

\begin{abstract}
Serum Homocysteine elevation, has been observed in some cerebral vinous sinus thrombosis patients. There is a complex overlap between homocysteine level and cerebrovascular disease, and the cause and significance of homocysteine rise in cerebral vinous sinus thrombosis are still controversial.To detect serum homocysteine level in patients with cerebral venous sinus thrombosis and its impact on its severity, short term outcome, size of thrombosis. In this case control study, sixteen patients of CVST (13 males \& 3 females) and 35 matched control subjects were recruited for this study. Serum homocysteine level was estimated by Enzyme-Linked Immunosorbent Assay (ELISA). Assessment of severity of thrombosis was done based on Canadian Neurological Scale. Assessment of outcome on discharge was done based on Barthel index score. In this study serum homocysteine level was raised in Cerebral Vinous Sinus Thrombosis cases when compared to controls on admission. The mean and standard deviation of homocysteine were $42.5 \pm 54.2 \mu \mathrm{mol} / \mathrm{l}$ in cases with significant $\mathrm{p}$ value of $<0.05$. Cases with high homocysteine levels had low severity scores, which indicate poor prognosis. Homocyteine elevation in cerebral vinous sinus thrombosis (CVST) patients was associated with CT changes, severe thrombosis, large thrombus size and bad outcome.
\end{abstract}

Key words: Homocysteine, Cerebral Venous Sinus Thrombosis.

\section{Introduction}

Cerebral venous sinus thrombosis (CVST) is an uncommon form of cerebrovascular stroke that mostly affects young adults and can be precipitated by a variety of conditions [1].

The clinical symptoms may vary from severe headache $(70-90 \%)$, focal lateralized signs $(25 \%$ $75 \%)$, seizures $(30-40 \%)$ as well as behavioral symptoms such as delirium, amnesia, and disturbances in consciousness. In some of the patients the clinical symptoms develop slowly whereas in a few the symptoms are non-specific requiring a high index of clinical suspicion to pick up the diagnosis. Therefore, it is not too unjustified that there does not exist a definite clinical syndrome [2].

There are many conditions predisposing to CVST, which may be seen alone or in combination. The known conditions are sinusitis, trauma, surgery, hypercoagulable states (antiphospholipid syndrome, protein $\mathrm{C}$ and protein $\mathrm{S}$ deficiency, anti-thrombin deficiency, vasculitis, pregnancy, puerperium, usage of OCPs, nephrotic syndrome, malignancy etc [3].

Homocysteine is an alpha amino acid which originated primarily from methionine which is a byproduct of protein metabolism. Under normal condition homocysteine is converted to cystathione and then to cysteine (via the transulfuration pathway requiring enzyme cystathione beta synthase) which is then excreted via the urinary route. Vitamin B6 is required as a co-factor in this pathway. Deficiency of the enzyme cystathione beta synthase thus causes homocysteinuria, an inborn error of metabolism. There is another pathway namely the remethylation pathway which requires methylene tetrahydrofolate reductase (from folate metabolism) which helps methionine synthetase to convert homocysteine back to methionine .Vitamin B 12 is a cofactor in this reaction and any pathology in this pathway leads to hyperhomocysteinemia [4].

Arterial vascular disease and lower limb deep vein thrombosis has a direct correlation with hyperhomocysteinemia and the strength of the correlation is well established. Many studies have also looked at the role of hyperhomocysteinemia in venous thromboembolism (VTE) [4].

Vascular occlusion, both arterial and venous, is the leading cause of morbidity and mortality in untreated patients with homocystinuria (HCU) due to cystathionine $\beta$-synthase (CBS) deficiency. The probability of suffering from a vascular event is $30 \%$ before the age of 20 years and rises to $50 \%$ before the age of 30 years in untreated patients [5]. Untreated patients have severe hyperhomocysteinaemia, hypermethioninaemia and hypocysteinaemia. Cerebral venous sinus thrombosis (CVST) is an uncommon but recognised complication of untreated HCU. CVST may present with non-specific clinical manifestations including headache, focal neurological deficits and seizures, and may follow a progressive course [6].

This study describes a patient with HCU, who developed seizures and an evolving left hemispheral syndrome secondary to CVST and discuss the role of homocysteine-lowering therapy and early anticoagulation in achieving a good clinical outcome in Benha, Egypt.

\section{Patients and methods}

This is a case control study, conducted from at the beginning of 2018 till April 2019 in Benha 
University Hospital. Study subjects were informed of the possibility of using the data obtained for academic purpose. Confidentiality was assured to all participants and data used for this study were stripped of personally identifiable information. They were also informed about their right to withdraw their consent at any point, without any consequence to them.

\subsection{Patients}

Study participants were in the age group of 25-83 yrs. Cerebral venous sinus thrombosis (CVST) patients $(n=16)$ and control subjects $(n=35)$ were selected by considering strict inclusion and exclusion criteria. We confirmed the thrombosis by computed tomography (CT) brain imaging. For comparison, age matched control subjects were selected. Excluded were the patients with prior impaired renal function (creatinine $>1.5 \mathrm{mg} / \mathrm{dl}$ ), S$\mathrm{T}$ elevation myocardial infarction and Patient unwilling to give informed consent.

\subsection{Methods}

All patients were subjected to the following: Thorough medical history taking, Full general and neurological examination, ECG, Blood sugar level, Kidney function test and coagulation profile.

Serum homocysteine level measurement: $5 \mathrm{ml}$ of venous blood will be obtained by venipuncture under aseptic conditions, centrifuged and the separated serum will be used for estimation. Serum homocysteine will be measured on admission. It will be measured by Enzyme-Linked Immunosorbent Assay (ELIZA), and will be considered abnormal if it was $\geq 15 \mu \mathrm{mol} / \mathrm{L}$

Patients' initial thrombosis severity was assessed on admission by the Canadian Neurological Scale,
Patients' thrombosis outcome was assessed on discharge by the Barthel ADL (activity of daily living) Index.

\subsection{Statistical analysis}

The collected data were tabulated and analyzed using SPSS version 16 software (Spss Inc., Chicago, ILL Company). Categorical data were presented as number and percentages while quantitative data were expressed as mean \pm standard deviation and range. Chi square test (X2), or Fisher's exact test (FET), were used to analyze categorical variables. Quantitative data were tested for normality using Kolmogorov-Smirnov test, and proved to be normally distributed, using Student, ANOVA and Person's correlation coefficient (r) for their analysis. The accepted level of significance in this work was stated at $0.05 \quad(\mathrm{P}<0.05$ was considered significant).

\section{Results}

In this study, $81.3 \%$ of the sample were males while $18.7 \%$ were females, with mean age of $54.6 \pm$ 17.7 years ranging from $25-83$ years. $18.7 \%$ of the patient group $(n=16)$ were non-smokers while $81.3 \%$ were smokers. $56.25 \%$ of the patients group were diabetic and $62.5 \%$ were hypertensive.

There is a statistical significant difference in laboratory results among the studied groups between the mean values of CRP \& D-dimer

This study shows that $56.25 \%$ of patients have increased serum homocysteine level; with mean 42.5 ranging from 93.5 to $170 \mu \mathrm{mol} / \mathrm{l}$. Serum homocysteine was normal in $43.7 \%$ of CVST patients while it was high in $56.3 \%$ of them, compared to controls who had normal Hcy levels. These results were highly significant.

Table (1) Homocysteine level in patient group

\begin{tabular}{llll}
\hline & & No. & \% \\
\hline \multirow{3}{*}{ Homocysteine } & Mormal & 7 & $43.75 \%$ \\
& Mild To Moderate High Levels & 7 & $43.75 \%$ \\
& Very High Levels & 2 & 12.5 \\
& Mean \pm SD & $42.5 \pm 54.2$ & \\
& Range & 93.5 to 170 \\
\hline
\end{tabular}

Table (2) Clinical symptoms of patients with cerebral venous sinus thrombosis.

\begin{tabular}{lll}
\hline Clinical symptoms & No. & $\%$ \\
\hline Headache & 10 & $62.5 \%$ \\
Table (2) Continue & & \\
\hline N/Vomiting & 6 & $37.5 \%$ \\
Seizure & 6 & $37.5 \%$ \\
Unconscious & 6 & $37.5 \%$ \\
Hemiparesis & 6 & $37.5 \%$ \\
Speech disturbance & 1 & $6.3 \%$ \\
Visual disturbance & 2 & $12.5 \%$ \\
\hline
\end{tabular}


This table shows that $62.5 \%$ of patients have headache, $37.5 \%$ have nausea and vomiting, $37.5 \%$ have generalized seizure, $37.5 \%$ have stupor or coma, $37.5 \%$ have motor hemiparesis, $6.3 \%$ have speech disturbance and $12.5 \%$ have visual disturbance.

Table (3) Location Of Thrombosis.

\begin{tabular}{lll}
\hline Location of thrombosis & No. of patients & \% \\
\hline Superior sagittal sinus (SSS) & 12 & $75.0 \%$ \\
Lateral sinus (LS) & 11 & $68.8 \%$ \\
BothSuperior sagittal sinus and Lateral & 7 & $43.8 \%$ \\
sinus (SSS+LS) & 4 & $25 \%$ \\
Straight sinus & 1 & $6.3 \%$ \\
Internal jugular vein & & \\
\hline
\end{tabular}

This table shows that the occluded sinus or cerebral vein was the superior sagittal sinus (SSS) in 12 cases (75.0\%), the lateral sinus (LS) in 11 cases $(68.8 \%)$, both sinuses in 7 cases $(43.8 \%)$, the straight sinus in 4 cases $(25.0 \%)$, and the internal jugular vein in 1 case $(6.3 \%)$.

Table (4) Laboratory results and risk factors in patient group

\begin{tabular}{lll}
\hline & No of patients & percentage \\
\hline D-dimer & 16 & $100 \%$ \\
Protein C deficiency & 0 & $0 \%$ \\
Protein S deficiency & 3 & $18.8 \%$ \\
Antithrombin deficiency & 2 & $12.5 \%$ \\
ANA & 0 & $0 \%$ \\
Anti-phospholipid syndrome & 0 & $0 \%$ \\
CRP at admission & 16 & $100 \%$ \\
Fibrinogen at admission & 14 & $87.5 \%$ \\
Anemia (Iron deficiency) & 3 & $18.8 \%$ \\
Hyperhomocysteinemia & 9 & $56.3 \%$ \\
Oral contraceptives (female n=3) & 1 & $33.3 \%$ \\
Table (4) Continue & & \\
\hline Nephrotic syndrome & 2 & $12.5 \%$ \\
Malignancy & 1 & $6.3 \%$ \\
Multiple sclerosis & 1 & $6.3 \%$ \\
Epilepsy & 1 & $6.3 \%$ \\
\hline
\end{tabular}

This table shows that D-dimer was increased in all patients with mean level $(5.61 \pm 4.39 \mathrm{ug} / \mathrm{ml})$; PT, INR and APTT were slightly decreased in 1 patient and in 5 patients, respectively. Protein S and antithrombin III activity were decreased in 3 patients and in 2 patients, respectively. Activity of protein $\mathrm{C}$ was normal in all patients. All patients showed negative or normal levels of anti-nuclear antibody and the antibodies related to antiphospholipid antibody syndrome. Three patients had iron deficiency anemia. CRP $(2.67 \pm 3.15 \mathrm{mg} / \mathrm{dl})$ and fibrinogen $(370 \pm 72 \mathrm{mg} / \mathrm{dl})$ levels were increased in 16 patients and 14 patients, respectively.

Table (5) The clinical and laboratory factors related to hyperhomocysteinemia

\begin{tabular}{llllllllll}
\hline Case & & & Hcy & FA & B12 & B6 & & OTHERS \\
No. & Age & Sex & & & & PAM & PAL & PIN & \\
\hline $\mathbf{1}$ & 78 & M & 14.6 & - & $/$ & $/$ & $/$ & $/$ & DM, Dementia \\
$\mathbf{2}$ & 81 & M & 13.6 & - & $/$ & $/$ & $/$ & $/$ & DM, Gastrectomy \\
$\mathbf{3}$ & 72 & M & 17.8 & - & $/$ & $/$ & $/$ & $/$ & - \\
$\mathbf{4}$ & 25 & M & 21.6 & - & $/$ & $/$ & $/$ & $/$ & MS, ATIIIdeficiency
\end{tabular}




\begin{tabular}{llllllllll}
$\mathbf{5}$ & 55 & M & 14.6 & 17.7 & 383 & 0.2 & 8.9 & 3.0 & Alcoholism, Smoking \\
$\mathbf{6}$ & 83 & F & 18.6 & 2.9 & 673 & $/$ & $/$ & 3.0 & - \\
7 & 54 & M & 18.1 & 6.1 & 304 & 0.2 & 2.3 & 3.0 & Multiple Myeloma \\
$\mathbf{8}$ & 36 & M & 170 & 1.1 & 178 & 0.2 & 7.5 & 3.0 & AED (epilepsy) \\
$\mathbf{9}$ & 63 & M & 93.5 & 2.4 & 152 & $/$ & $/$ & $/$ & Alcoholism, Smoking \\
\hline
\end{tabular}

*Hcy: Homocysteine (normal values: $3.7-13.5 \mu \mathrm{mol} / \mathrm{L}$ ).

*DM: Diabetes Mellitus. *AED: Antiepileptic Drug.

*FA: Folate (normal values: 3.6-12.9 ng/mL). *B12: Vitamin B12 (normal values: 180-914 pg/mL).

*PAM: Pyridoxamine (normal values: <0.6 ng/mL). *PAL: Pyridoxal (normal values: 6.0-40.0 ng/mL).

*PIN: Pyridoxine (normal values: $<3.0 \mathrm{ng} / \mathrm{mL}$ ). *MS: Multiple Sclerosis.

*AT: Antithrombin.

This table shows that Hyper-Hcy was identified in 9 (56.3\%) of the 16 patients. Seven patients (Case 1 7) had slightly to moderately high levels (13.6-21.6 $\mu \mathrm{mol} / \mathrm{l}$; normal: $3.7-13.5 \mu \mathrm{mol} / \mathrm{l})$, and 2 patients (Case 8 and 9) had very high levels (170 and $93.5 \mu \mathrm{mol} / \mathrm{l})$. Furthermore, in 9 patients with hyper-Hcy, 1 (Case 6) had folate deficiency, and 1 (Case 7) had vitamin B6 deficiency. And 2 patients (Case 8 and 9) with very high levels of Hcy showed combined deficiencies of folate and vitamin B12.

Table (6) Comparing mean values of Serum Homocysteine level among the studied patients according to sociodemographic characters.

\begin{tabular}{lllllll}
\hline & & \multicolumn{2}{l}{ Normal (No.=7) } & \multicolumn{2}{l}{ Increased (No.=9) } & \\
& & No. & \% & No. & \% & P- \\
\hline \multirow{3}{*}{ Sex } & Female & 2 & $28.5 \%$ & 1 & $11.1 \%$ & \\
& Male & 5 & $71.4 \%$ & 8 & $88.8 \%$ & 0.099 \\
& Non-smoker & 2 & $28.5 \%$ & 6 & $75.0 \%$ & \\
Age & Smoker & 5 & $71.4 \%$ & 2 & $25.0 \%$ & \\
& Mean \pm SD & $44.5 \pm 9.30$ & $60.8 \pm 20.3$ & \\
& Range & $35-55$ & $40-81$ & 0.525 \\
\hline
\end{tabular}

This table shows that difference of Homocysteine levels among the studied patients according to age and smoking is statistically insignificant $(\mathrm{P}>0.05)$, while this difference is statistically significant according to $\operatorname{sex}(\mathrm{P}<0.05)$.

Table (7) Comparing mean values of Serum Homocyteine level among the studied patients according to comorbidity:

\begin{tabular}{|c|c|c|c|c|c|c|}
\hline & & \multicolumn{2}{|c|}{ Increased $($ No. $=9)$} & \multicolumn{2}{|c|}{ Normal (No.=7) } & \multirow[t]{2}{*}{ P-value } \\
\hline & & No. & $\%$ & No. & $\%$ & \\
\hline \multirow[t]{2}{*}{$\overline{\mathbf{D M}}$} & Negative & 7 & $77.7 \%$ & 0 & $0.0 \%$ & 0.263 \\
\hline & Positive & 2 & $22.2 \%$ & 7 & $100 \%$ & \\
\hline HTN & $\begin{array}{l}\text { Negative } \\
\text { Positive }\end{array}$ & $\begin{array}{l}3 \\
6\end{array}$ & $\begin{array}{l}33.3 \% \\
66.6 \%\end{array}$ & $\begin{array}{l}3 \\
4\end{array}$ & $\begin{array}{l}42.8 \% \\
57.1 \%\end{array}$ & 0.613 \\
\hline
\end{tabular}

This table shows that $67 \%$ of patients with high Homocyteine level are hypertensive and $22 \%$ of them were diabetic compared to $43 \%$ of patients with normal Homocyteine level who don't have HTN but this difference was statistically non-significant $(\mathrm{P}>0.05)$.

Table (8) Homocysteine level among the studied patients according to disease characters

\begin{tabular}{lllll}
\hline Location Of Thrombosis & \multicolumn{2}{l}{ Increased HCY (No.=9) } & $\begin{array}{l}\text { Normal HCY } \\
\text { (No.=7) }\end{array}$ & $\begin{array}{l}\text { P- } \\
\text { value }\end{array}$ \\
& No. & $\%$ & No. $\%$ & 0.604 \\
Thrombosis in SSS (superior & 6 & 66.7 & 5 & $71.4 \%$ \\
sagittal sinus) & & $\%$ & &
\end{tabular}

This table shows there is a statistically significant increase in outcome among patients with high homocysteine level. 


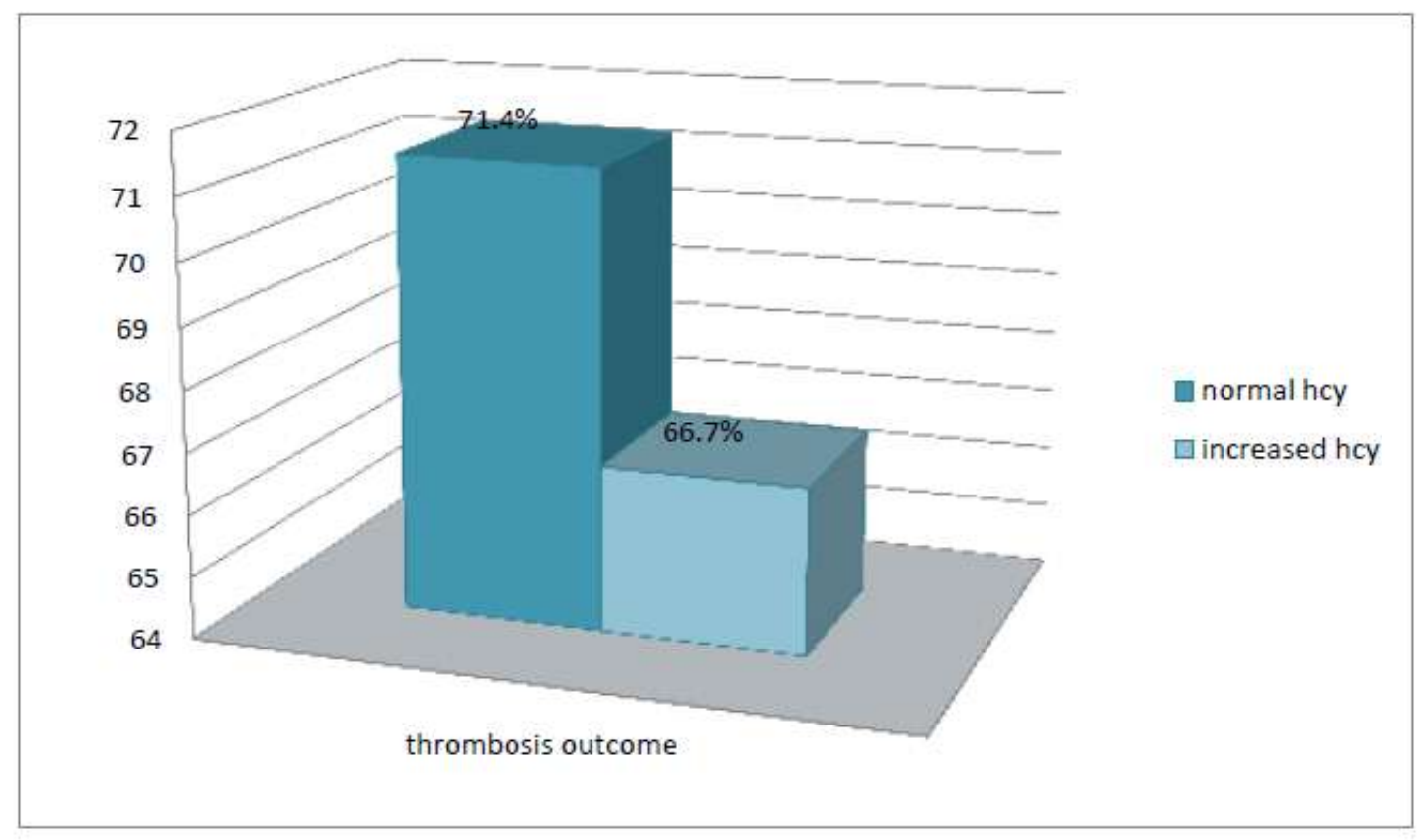

Fig (1) Comparison of Serum thrombosis outcome among the studied patients according to homocysteine level

\section{Discussion}

Patients with CVT have an occlusion of one or more of the dural sinuses of the brain, often in combination with cortical vein thrombosis [7].

A small proportion of patients only have an occlusion of a cortical vein, which is termed isolated cortical vein thrombosis. CVT leads to a diminished outflow of blood and cerebrospinal fluid, which in about $50 \%$ of patients results in development of a venous infarct. In contrast to arterial infarcts, CVT mostly affects young adults and children and it is an important cause of stroke in the young [7].

This is a comparative cross-sectional nonrandomized study made in Neuropsychiatry department at Benha University Hospital and Air Force Hospital aimed to detect serum Homocysteine level in patients with cerebral venous sinus thrombosis and its impact on its severity, short term outcome, location of the thrombus. The studied sample was divided to 16 patients and 35 controls.

In the current study, $56.3 \%$ of cerebral venous sinus thrombosis patients had elevated serum Homocysteine level, which was congruent with previous studies. Associations, causes and value of Homocysteine rise have been the core of many studies. It has been demonstrated that Homocysteine elevation could be associated with more severe thrombosis, specific thrombus locations, laboratory results and poor outcome [8]. In this study, $56.3 \%$ of patient had increased serum homocysteine, the mean of patient was 42.5 with range from 93.5 to $170 \mu \mathrm{mol} / \mathrm{l}$.

In addition, the mean age of the studied sample was
$(54.6 \pm 17.7)$ years for CVT patients and controls. The number of female patients with CVT in our study was one patient $(11.1 \%)$ compared to 8 male patients $(88.9 \%)(66.6 \%)$ ( of patients with high homocysteine level are hypertensive and $22.2 \%$ of them were diabetic compared to none of patients with normal homocysteine level who don't have DM, and 42.8\% who don't have HTN but this difference was statistically non-significant.

This study revealed that there is a statistically significant increase CVT outcome among patients with high homocysteine levels.

The most common risk factor in women has been reported to be oral contraceptive use. The incidence of CVT in our study was $0.23 \%$ of all kinds of strokes or $0.30 \%$ of ischemic infarctions, which may be lower than previously reported. This may be due to few young female CVT patients using oral contraceptives.

The incidence of clinical symptoms was variable; there was an $81 \%$ to $98 \%$ incidence of headache, a $27-76 \%$ incidence of seizure, a $10-64 \%$ incidence of unconsciousness, and a $27 \%$ to $76 \%$ incidence of focal neurological deficits. Headache is the most common symptom in CVT. In ISCVT, CVT patients without headache were frequently older male patients. This study showed a low incidence of headache, $62.5 \%$, which was due to the high percentage of older men in our study.

Saadatnia M et al, (2009), proposed that CVT in the past was attributed to infections of the face and otomastoid areas, but after the introduction of antibiotics, it is more often related to pregnancy, puerperium, neoplasms, dehydration, genetic or acquired thrombophrenia, and oral contraceptives. 
Especially in Western countries, CVT is increased by the hypercoagulability induced by oral contraceptive use [9].

In this study, the patients with CVT had as risk factors oral contraceptive use (1 patient), deficiencies of protein $\mathrm{S}$ (3 patients) and antithrombin III ( 2 patients), nephrotic syndrome (2 cases), iron deficiency anemia (3 patients), colon cancer (1 patient), and multiple myeloma in remission (1 patient). Furthermore, hyper-Hcy was identified in $9(56.3 \%)$ of 16 patients.

Lauw MN et al, (2013) revealed that metabolism of Hcy is regulated by 2 major pathways remethylation and transsulfuration. Folate, vitamin B12 and Vitamin B6 play roles through the conversion or catalytic process in these pathways. Thus, the deficiencies of these vitamins induce hyper-Hcy. Furthermore, environmental factors also have an important influence on the level of serum Hcy. Raised levels are found in some chronic diseases such as DM, and malignancy; after the administration of certain drugs such as oral contraceptives, AEDs, and methotrexate; and in patients of older age, male sex, postmenopausal status, heavy smoking history, etc. Folate and vitamin deficiencies can also occur because of chronic alcoholism, or low intake and disturbed digestive absorption of green or yellow vegetables and other foods containing these vitamins [10].

In this study, There were 9 cases of hyper-Hcy. There was 1 case of folate deficiency, 1 case of B6 deficiency, and 2 cases of combined deficiencies of B12 and folate. One patient had total resection of the stomach, 2 had chronic alcoholism, 2 were heavy smokers, 2 had DM, and 1 was taking an AED. These disorders and habits may increase the level of Hcy. Additionally, it should be recognized that even acquired conditions such as vitamin deficiencies, especially the deficiencies of both folate and vitamin B12, increased the level of Hcy to more than $100 \mu \mathrm{mol} / \mathrm{l}$.

The most frequent complications observed in patients with hyper-Hcy in this study are thrombotic diseases . Martinelli I et al, (2003) supposed that arterial and venous thromboses are potential complications and confirm that HyperHcy is a risk factor for CVT, and its risk ratio is 4fold compared to controls. So in this study hyperHcy is identified in $56.3 \%$ of CVT patients, the prevalence of hyper-Hcy as a risk factor for CVT could be high [11].

Hyper-Hcy is a treatable risk factor in CVT and can be corrected by the supplementation of deficient vitamins and changes in lifestyle. It should be payed attention to hyper-Hcy as a risk factor for CVT [11].

So, CVT commonly occurred in older males. The prevalence of hyper- Hcy as a risk factor of CVT was high, and the main underlying disorders were folate and vitamin B12 or B6 deficiencies. This is clinically important, because these acquired risks can be corrected by supplementation therapy to prevent the recurrence of CVT [11].

\section{Conclusion}

5.1We concluded the following from this study The relationship of serum homocysteine level to cerebral venous sinus thrombosis is controversial, the present results revealed that:

There is significant increase in serum homocysteine level within CVST patients.

Severe thrombosis, large thrombus size and bad outcome was associated with high serum homocysteine level.

A significant positive correlation between laboratory results and homocysteine level in patient group.

6. Limitations of the stud:

6.1 Limitations met while conducting this study were:

This is not a population-based study. It is a singlecenter case control study restricted to a local hospital, based on the data collected from a small number of ethnically homogeneous, unselected clinically assessed CVST patients, therefore may not accurately reflect the majority of other population.

\section{Recommendations}

Taking into account, all the limitations of the study and the previous literature on the prevalence of cerebral venous sinus thrombosis (CVST), we strongly recommend that:

Patients with homocysteine elevation could not be attributed to renal or cardiac problem, and neurogenic vascular injury could be suspected.

There are some CVST patients with possible neurogenic vascular injury, it is prudent to be vigilant in those with high homocysteine and perform appropriate cardiac, renal and coagulation evaluation.

Individuals with risk factors of CVST such as hypertension, diabetics abnormal coagulated factors should be monitored for homocysteine level.

\section{References}

[1] W.Ageno, C.Becattini, T.Brighton, R.Selby, P.W.Kamphuisen, Cardiovascular risk factors and venous thromboembolism: a meta- analysis. Circulation, Vol.117, PP. 93102,2008 .

[2] T.A.Ozcan, H.Meral, S.Ozben, R.Tiras, H.Hakyemez, O.Ozturk, A.Mutlu, B.P.Balci, O.Cokar and F.Ozer, Cerebral venous sinus thrombosis: gender differences in ten years' experience. Düşünen Adam: The Journal of Psychiatry and Neurological Sciences, Vol.26 (3), PP.281-258,2013.

[3] M.Den Heijer, T.Koster, H.J.Blom, G.M.Bos, E.Briet, P.H.Reitsma, 
J.P.Vandenbroucke, $\quad$ F.R.Rosendaal, Hyperhomocysteinemia as a Risk Factor for Deep-Vein Thrombosis. N Engl J Med,Vol. 334, PP.759-762, 1996،

[4] P.Simioni, P.Prandoni, A.Burlina, Tormene, C.Sardella, V.Ferrari, L.Benedetti, A.Girolami, Hyperhomocysteinemia and deepvein Thrombosis. A case-control study. Thromb Haemost,Vol.76, PP.883-6,1996.

[5] S.H.Mudd, F.Skovby, H.L.Levy, The natural history of homocystinuria due to cystathionine $\beta$-synthase deficiency. Am J Hum Genet, Vol.37, PP. 1-31, 1985.

[6] F.B.Cochran and S.Packman, Homocystinuria presenting as sagittal sinus thrombosis. Eur Neurol,Vol.32, PP. 13,1992.

[7] J.M.Coutinho, Cerebral venous thrombosis. J Thromb Haemost ,Vol.13, PP. 238244,2015.

[8] J.M.Ferro, P.Canhao, J.Stam, M.G.Bousser, F.Barinagarrementeria, Investigators ISCVT, Prognosis of cerebral vein and dural sinus thrombosis: results of the International Study on Cerebral Vein and Dural Sinus Thrombosis (ISCVT). Stroke ,Vol.35, PP.664-670,2004.

[9] M.Saadatnia, F.Fatehi, K.Basiri, Cerebral venous sinus thrombosis risk factors. Int $\mathrm{J}$ Stroke ,Vol.4, PP.111-123,2009.

[10] M.N.Lauw, S .Barco, J.M.Coutinho, S.Middeldorp, Cerebral venous thrombosis and thrombophilia: a systematic review and metaanalysis. Semin Thromb Hemost,Vol.39, PP.913-927,2013.

[11] Martinelli, T.Battaglioli, P.Pedotti, Hyperhomocysteinemia in cerebral vein throm bosis. 